\title{
An unusual Actinobacillus equuli strain isolated from a rabbit with Tyzzer's disease
}

\author{
H. Moyaert*, A. Decostere, M. Baele, K. Hermans, P. Tavernier, \\ K. Chiers, F. Haesebrouck \\ Department of Pathology, Bacteriology and Avian Diseases, Faculty of Veterinary Medicine, \\ Ghent University, Salisburylaan 133, B-9820 Merelbeke, Belgium
}

Received 8 January 2007; received in revised form 30 March 2007; accepted 4 April 2007

\begin{abstract}
Actinobacillus equuli was isolated in pure culture from the liver and lungs of an adult rabbit with Tyzzer's disease (Clostridium piliforme). Based on the haemolytic features on blood agar plates, a positive reaction in the CAMP-test, hydrolysis of esculin, the inability to ferment L-arabinose, tDNA-PCR and sequencing of the 16S rRNA gene, the isolate was classified as $A$. equuli subsp. haemolyticus biovar 1 . However, the aqxA gene, characteristic for haemolytic A. equuli strains, was not detected by PCR.

(C) 2007 Elsevier B.V. All rights reserved.
\end{abstract}

Keywords: Actinobacillus equuli; Rabbit

Actinobacillus equuli, a member of the family Pasteurellaceae, is a small, pleomorphic, non-motile Gram-negative rod. It is the etiological agent of a rare, but frequently fatal septicaemia in neonatal foals also known as "sleepy foal disease" which is spread worldwide (Rycroft and Garside, 2000; Berthoud et al., 2002). Besides this acute form of disease, $A$. equuli can be associated with other types of infections in both foals and adult horses, such as abortion, enteritis, peritonitis, pleuropneumonia, pericarditis, periorchitis and arthritis (Webb et al., 1976; Dill et al., 1982; Al-Mashat and Taylor, 1986; Belknap et al.,

\footnotetext{
* Corresponding author. Tel.: +329 2647434; fax: +329 2647494.

E-mail address: Hilde.Moyaert@UGent.be (H. Moyaert).
}

1988; Peremans et al., 1991; Collins et al., 1994; Patterson-Kane et al., 2001). A. equuli has been subdivided in two different subspecies, designated $A$. equuli subsp. haemolyticus (formerly described as Bisgaard Taxon 11), which is haemolytic and enhances the haemolytic effect of staphylococcal $\beta$ haemolysin (CAMP-positive) and A. equuli subsp. equuli, which is non-haemolytic and CAMP-negative (Christensen et al., 2002). The haemolytic strains have mainly been described in horses, but are not specifically associated with "sleepy foal disease", which is typically linked with the non-haemolytic strains (Kuhnert et al., 2003). A. equuli subsp. equuli was not only isolated from horses, but has also been associated with abortion and endocarditis in pigs 
(Jones and Simmons, 1971; Werdin et al., 1976). Moreover, the organism was isolated from the nasopharynx, conjunctiva and middle ear of guinea pigs, rats and mice (Lentsch and Wagner, 1980). A. equuli is additionally considered as a potential opportunistic pathogen of humans, especially in immunocompromised people or people who are occupationally exposed to infected horses or pigs (Ashhurst-Smith et al., 1998; Vaz et al., 2001).

In this report, we describe the isolation of an unusual A. equuli strain from the liver and lungs of an adult rabbit with Tyzzer's disease.

An adult, male pet-rabbit (Oryctolagus cuniculus) weighing $4.1 \mathrm{~kg}$, died after a 4-day history of anorexia and depression. The animal had not been treated with an antimicrobial agent.

At necropsy, a fibrinous pericarditis as well as numerous 1-mm-diameter necrotic foci scattered within the liver parenchyma were observed. Histological examination of haematoxylin-eosin stained sections of formaldehyde (10\%) fixed, paraffin embedded liver tissue samples revealed a focal extensive necrotizing hepatitis. In Warthin-Starrystained sections of the liver, numerous large, elongated bacilli were observed within the cytoplasm of viable hepatocytes at the margins of necrotic foci. The latter finding is diagnostic for Tyzzer's disease, a rapidly progressing, acute hepatitis caused by Clostridium piliforme (Ganaway et al., 1971; Kelly, 2000).

Liver and lung samples of the animal were bacteriologically examined. Cultures of these samples on Columbia agar with 5\% sheep blood (Oxoid, Basingstoke, UK) yielded profuse and pure growth of haemolytic bacteria which produced greyish-white colonies. Gram staining revealed small, pleomorphic Gram-negative rods. The organism was oxidase, catalase, urease and CAMP-positive, but negative for L-arabinose fermentation. In a bile containing medium, hydrolysis of esculin was observed. Based on these phenotypic characteristics, the isolate was identified as A. equuli subsp. haemolyticus biovar 1 (Christensen et al., 2002).

Using tDNA-PCR, the organism showed an identical pattern to A. equuli and A. suis (Baele et al., 2000). Sequencing of the 16S rRNA gene (Baele et al., 2001) yielded a fragment of $1214 \mathrm{bp}$ (accession number: EF141826) which showed $99 \%$ similarity to A. suis and A. equuli subsp. haemolyticus and $98 \%$ similarity to A. equuli subsp. equuli. Subsequently, $a q x \mathrm{~A}, a p x \mathrm{ICA}$ and apxIICA PCR-assays were carried out (Frey et al., 1995; Berthoud et al., 2002). In these PCR-assays, A. equuli subsp. haemolyticus CCUG $19799^{\mathrm{T}}$, an A. suis field isolate and A. pleuropneumoniae serotypes 1-12 reference strains were used as controls. None of these PCR-assays yielded an amplicon with DNA of the rabbit isolate, while the expected amplicons were obtained with DNA of the control strains (Frey et al., 1995; Kuhnert et al., 2003).

Within the genus Actinobacillus, haemolysis is observed for A. pleuropneumoniae, A. equuli subsp. haemolyticus and $A$. suis. In contrast with $A$. pleuropneumoniae and most of the A. equuli strains, all A. suis strains have the ability to ferment Larabinose. However, these closely related species cannot unequivocally be separated based on phenotypic characteristics alone. Additionally, 16S rRNA gene sequence comparison and tDNA-PCR are not able to separate A. equuli subsp. equuli, A. equuli subsp. haemolyticus and A. suis at the (sub)species level. Therefore, PCR typing for RTX (repeat in toxin) genes is recommended (Kuhnert et al., 2003; Christensen and Bisgaard, 2004). Indeed, A. equuli subsp. equuli does not contain apxICA, apxIICA nor aqxA genes. In contrast, A. equuli subsp. haemolyticus strains contain the $a q x \mathrm{~A}$ gene and neither apxICA nor apxIICA genes, while A. suis isolates contain apxICA and apxIICA but no aqxA genes (Kuhnert et al., 2003). A. pleuropneumoniae serotypes 1, 5a, 5b, 9, 10, 11 and 14 contain the apxICA gene and apxIICA is present in all serotypes except in serotypes 10 and 14 (Frey et al., 1995; Schaller et al., 2001).

Phenotypically, the rabbit isolate was identified as $A$. equuli subsp. haemolyticus biovar 1. Contradictorily, since we could not detect amplicons of the aqxA gene, our isolate would be identified as A. equuli subsp. equuli. The haemolytic activity of our isolate and its ability to enhance the haemolytic effect of staphylococcal $\beta$-haemolysin indicate the presence of an RTXtoxin (Devenish et al., 1992). The negative result in the aqxA-specific PCR might be caused by a DNAmutation in one or both primer binding sites, resulting in failure of primer annealing and amplification. Alternatively, one might speculate on the presence of a different RTX-toxin in this rabbit-isolate.

Our finding that this A. equuli strain was isolated in pure culture from both the lungs and the liver might 
suggest that it contributed to the disease, probably as a secondary invader. Recently, a comparable case has been reported by Meyerholz and Haynes (2005): a 5 -year-old pet rabbit died after a 3-day history of anorexia and depression. This rabbit suffered from an Actinobacillus capsulatus septicaemia, secondary to the gastric stasis syndrome ("wool block"). Veterinarians and diagnosticians need to be aware of Actinobacillus species as a potentially under-diagnosed bacterial pathogen of lagomorphs, especially since Actinobacillus spp. may be misidentified as Pasteurella spp., which are quite common in rabbits (Lentsch and Wagner, 1980; DeLong and Manning, 1994).

\section{Acknowledgments}

We are grateful to Serge Verbanck and Arlette Van de Kerckhove for their technical assistance.

\section{References}

Al-Mashat, R.R., Taylor, D.J., 1986. Bacteria in enteric lesions of horses. Vet. Rec. 118, 453-458.

Ashhurst-Smith, C., Norton, R., Thoreau, W., Peel, M.M., 1998. Actinobacillus equuli septicaemia: an unusual zoonotic infection. J. Clin. Microbiol. 36, 2789-2790.

Baele, M., Baele, P., Vaneechoutte, M., Storms, V., Butaye, P., Devriese, L.A., Verschraegen, G., Gillis, M., Haesebrouck, F., 2000. Application of tRNA intergenic spacer PCR for identification of Enterococcus species. J. Clin. Microbiol. 38, 4201-4207.

Baele, M., Chiers, K., Devriese, L.A., Smith, H.E., Wisselink, H.J., Vaneechoutte, M., Haesebrouck, F., 2001. The gram-positive tonsillar and nasal flora of piglets before and after weaning. J. Appl. Microbiol. 91, 997-1003.

Belknap, J., Arden, W., Yamini, B., 1988. Septic periorchitis in a horse. J. Am. Vet. Med. Assoc. 192, 363-364.

Berthoud, H., Frey, J., Kuhnert, P., 2002. Characterization of Aqx and its operon: the haemolytic RTX determinant of Actinobacillus equuli. Vet. Microbiol. 87, 159-174.

Christensen, H., Bisgaard, M., 2004. Revised definition of Actinobacillus sensu stricto isolated from animals. A review with special emphasis on diagnosis. Vet. Microbiol. 99, 13-30.

Christensen, H., Bisgaard, M., Olsen, J.E., 2002. Reclassification of equine isolates previously reported as Actinobacillus equuli, variants of A. equuli, Actinobacillus suis or Bisgaard taxon 11 and proposal of A. equuli subsp. equuli subsp. nov. and $A$ equuli subsp. haemolyticus subsp. nov. Int. J. Syst. Evol. Microbiol. 52, 1569-1576.

Collins, M.B., Hodgson, D.R., Hutchins, D.R., 1994. Pleural effusion associated with acute and chronic pleuropneumonia and pleuritis secondary to thoracic wounds in horses: 43 cases (1982-1992). J. Am. Vet. Med. Assoc. 205, 1753-1758.

DeLong, D., Manning, P.J., 1994. Bacterial diseases. In: Manning, P.J., Ringler, D.H., Newcomer, C.E. (Eds.), The Biology of Laboratory Rabbit. Academic Press, San Diego, pp. 129-170.

Devenish, J., Brown, J.E., Rosendal, S., 1992. Association of the RTX proteins of Actinobacillus pleuropneumoniae with haemolytic, CAMP, and neutrophil-cytotoxic activities. Inf. Immun. 60, 2139-2142.

Dill, S.G., Simoncini, D.C., Bolton, G.R., Rendano, V.T., Crissman, J.W., King, J.M., Tennant, B.C., 1982. Fibrinous pericarditis in the horse. J. Am. Vet. Med. Assoc. 180, 266-271.

Frey, J., Beck, M., van den Bosch, J.F., Segers, R.P.A.M., Nicolet, J., 1995. Development of an efficient PCR method for toxin typing of Actinobacillus pleuropneumoniae strains. Mol. Cell. Probes 9, 277-282.

Ganaway, J.R., Allen, A.M., Moore, T.D., 1971. Tyzzer's disease of rabbits: isolation and propagation of Bacillus piliformis (Tyzzer) in embryonated eggs. Infect. Immun. 3, 429-437.

Jones, J.E., Simmons, J.R., 1971. Endocarditis in the pig caused by Actinobacillus equuli: a field and an experimental case. Br. Vet. J. 127, 25-29.

Kelly, A., 2000. Tyzzer's disease in a 11-day-old foal. Can. Vet. J. 41, 491-492.

Kuhnert, P., Berthoud, H., Christensen, H., Bisgaard, M., Fey, J., 2003. Phylogenetic relationship of equine Actinobacillus species and distribution of RTX toxin genes among clusters. Vet. Res. 34, 353-359.

Lentsch, R.H., Wagner, J.E., 1980. Isolation of Actinobacillus lignieresii and Actinobacillus equuli from laboratory rodents. J. Clin. Microbiol. 12, 351-354.

Meyerholz, D.K., Haynes, J.S., 2005. Actinobacillus capsulatus septicemia in a domestic rabbit (Oryctolagus cuniculus). J. Vet. Diagn. Invest. 17, 83-85.

Patterson-Kane, J.C., Donahue, J.M., Harrison, L.R., 2001. Septicaemia and peritonitis due to Actinobacillus equuli infection in an adult horse. Vet. Pathol. 38, 230-232.

Peremans, K., Verschooten, F., De Moor, A., Desmet, P., 1991. Monoarticular infectious arthritis in the horse: 34 cases. J. Eq Vet. Sci. 11, 27-32.

Rycroft, A.N., Garside, L.H., 2000. Actinobacillus species and their role in animal disease. Vet. J. 159, 18-36.

Schaller, A., Djordjevic, S.P., Eamens, G.J., Forbes, W.A., Kuhn, R., Kuhnert, P., Gottschalk, M., Nicolet, J., Frey, J., 2001. Identification and detection of Actinobacillus pleuropneumoniae by PCR based on the gene apxIVA. Vet. Microbiol. 79, 47-62.

Vaz, R., Kennedy, K., Peel, M., 2001. Septicemia in a horse trainer caused by Actinobacillus equuli. Clin. Microbiol. Newsl. 23, 151-153.

Webb, R.F., Cockram, F.A., Pryde, L., 1976. The isolation of Actinobacillus equuli from equine abortion. Aust. Vet. J. 52, 100-101.

Werdin, R.E., Hurtgen, J.P., Bates, F.Y., Borgwardt, F.C., 1976. Porcine abortion caused by Actinobacillus equuli. J. Am. Vet. Med. Assoc. 169, 704-706. 